Proceedings

\title{
Design, Modeling, and Characterization of a Bionically Inspired Integrated Micro-Flapper for Cooling and Venting Applications ${ }^{+}$
}

\author{
Regine Behlert 1,*, Matthias Gehring ${ }^{1}$, Hannes Mehner ${ }^{2}$, Robert Wieland ${ }^{3}$ and Gabriele Schrag 1 \\ 1 Institute for Physics of Electrotechnology, Technical University of Munich, 80333 München, Germany; \\ gehring@tep.ei.tum.de (M.G.); schrag@tep.ei.tum.de (G.S.) \\ 2 Micromechanical Systems Group, Technical University Ilmenau, 98693 Ilmenau, Germany; \\ hannes.mehner@gmx.de \\ 3 Fraunhofer-Einrichtung für Mikrosysteme und Festkörper-Technologien EMFT, Munich, Germany; \\ robert.wieland@emft.fraunhofer.de \\ * Correspondence: behlert@tep.ei.tum.de; Tel.: +49-89-289-23109 \\ + Presented at the Eurosensors 2017 Conference, Paris, France, 3-6 September 2017.
}

Published: 23 August 2017

\begin{abstract}
A novel, integrated micromechanical actuator based on the principle of undulatory fish locomotion and intended for efficient on-chip cooling or venting applications is conceived and designed for an aluminum nitride-based thin-film technology applying coupled piezoelectric, mechanical, and fluidic analyses. The results of the investigations prove that an undulatory motion of the fin-like micro-flapper yields a significant higher, directed fluidic net flow compared to resonant-like motion. First prototypes have been manufactured and characterized applying laser Doppler vibrometry (LDV).
\end{abstract}

Keywords: piezoelectricity; fluidic actuator; laser doppler vibrometry; thin-film technology

\section{Device Idea and Bionical Approach}

The aim of this project is to design, manufacture and test a micromechanical actuator for efficient fluidic transport. To transfer momentum from the solid actuator to the fluid we created a flexible finshaped structure with a large aspect ratio (Figure 1b), which performs a motion adapted from the socalled undulation - the predominant pattern of movement of fish locomotion (respectively insects' flight) in nature.

To move forward the body of a fish performs a wavelike motion with increasing amplitude towards the tail fin [1]. This motion can be regarded as a superposition of a translatory motion during the up- and down-stroke of the fin and a rotational motion at the transition points of the oscillation, providing an adequate angle of attack between the fin and the fluid for efficient locomotion [2]. This sequence generates vortices with a well-defined sense of rotation at the transition points, forming a vortex wake behind the animal with a strong axial jet stream at its center (Figure 1a). The consequential transfer of momentum from the body to the fluid results in the forward motion of the fish, respectively the desired volume flow behind the flapping actuator. 


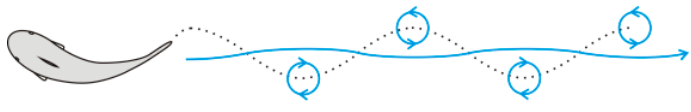

(a)

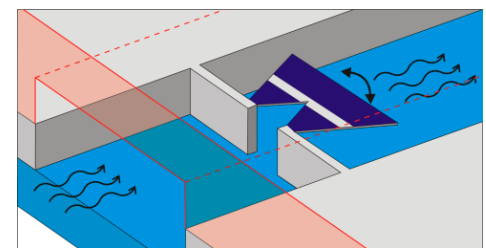

(b)

Figure 1. (a) The superposition of the vortices generated at the transition points of the oscillating tail fin results in an axial jet stream behind the swimming fish. (b) Schematic micro flapper mounted inside a micro channel: By undulatory up- und down-motion a directed air flow is generated.

\section{Technological Realization and Measurements of Prototypes}

The device is driven by a piezoelectric monomorph structure, using an aluminum nitride (AlN) thin-film, which is sputtered on top of a silicon layer (Si) with comparable thickness (based on [3]). Exploiting the piezoelectric $\mathrm{d}_{31}$-effect, the structure bends in vertical direction, when an electric field is applied in vertical direction. Devices were realized with thicknesses of $1 \mu \mathrm{m}$ to $3 \mu \mathrm{m}$ and lengths between $300 \mu \mathrm{m}$ and $1500 \mu \mathrm{m}$. Optical measurements revealed surprisingly high out of plane deflections (5-10 $\mu \mathrm{m}$, even up to $34 \mu \mathrm{m}$ at an actuator length of $300 \mu \mathrm{m})$ when applying sinusoidal voltages of $10 \mathrm{~V}$ at the resonant frequencies of the devices.

In this first process run simplified one-electrode designs (Figure 2a) were manufactured, i.e., not undulatory excitation is possible, yet. These samples are intended for calibration of the piezoelectricmechanically coupled finite element simulations of the pursued two-electrode design. The conclusions from this run deliver valuable information for the second process run and for potential re-design.

Measurements of the fundamental eigenfrequency are used to extract the length of the underetched part of the fixing bar (Figure 2d). This is a parasitic effect, which for future prototypes is to be avoided by choosing a more suitable wafer design, but it has a strong influence on the eigenfrequencies of this first set of prototypes, hence it is implemented in the current mechanical simulations. Second, the piezoelectric coupling coefficient $\mathrm{d}_{31}$ is extracted from the step response of the device (Figure 2c). It differs from its macroscopic value, due to the applied thin-film technology, because it strongly depends on the crystalline quality of the grown AlN layer. Decay curves of the step response (Figure $2 \mathrm{~b}$ ) are used to extract a damping coefficient, which is fed into a simple but sufficient damping model of a linearly damped spring mass system. The determined values show a strong dependency on the actuation voltage, hence the amplitude of the deflection. Consequently, this simplified model can only be applied for linearized operation around specific working points, which is, however, sufficient for the re-design of the devices.

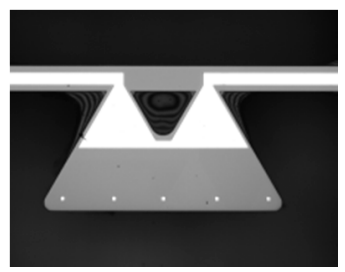

(a)

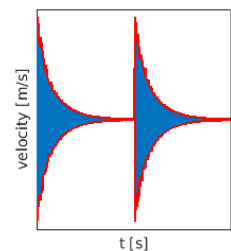

(b)

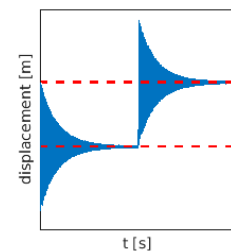

(c)

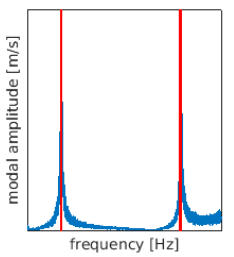

(d)

Figure 2. (a) Top view of a $300 \mu \mathrm{m}$ long and $3.1 \mu \mathrm{m}$ thick twin-fin flapper with a one electrode design. (b-d) Calibration of the piezoelectric mechanically coupled simulations by: (b) Decay curves from the step response to extract the damping coefficient; (c) static deflection of the flapper to extract $\mathrm{d}_{31}$ of the AlN layer and (d) measurement of the resonant frequencies.

\section{Calibrated Piezoelectric Mechanical Simulations}

An exemplary twin-fin flapper (Figure 2a) is simulated based on the calibrations described in the previous paragraph. The principal model is depicted in Figure 3a. Due to technological reasons $62 \%$ of the originally solid fixation bar is underetched, resulting in a quasi-spring-like suspension. 
The flapper is clamped at the left side of the bar, while symmetry boundary conditions are applied to the middle of the device. An electrical potential is applied to the electrode (green area in Figure $3 a)$, while the AlN/Si interface is electrically grounded. The measurement of the $d_{31}$ coefficient of the AlN resulted in a material quality of $80 \%$ in comparison to the established macroscopic value. The damping coefficient is implemented as a linear damping force acting on the surface of the flapper and depends on the oscillating mass of the device and its current velocity.

As shown in Figure $3 \mathrm{~b}$ the simulated frequency response shows good agreement with the corresponding measurements. In particular, the knowledge of the damping force is inevitable for the re-design process and the evaluation of different design variants, since no reliable predictions on the reachable amplitudes of deflection have been possible so far.

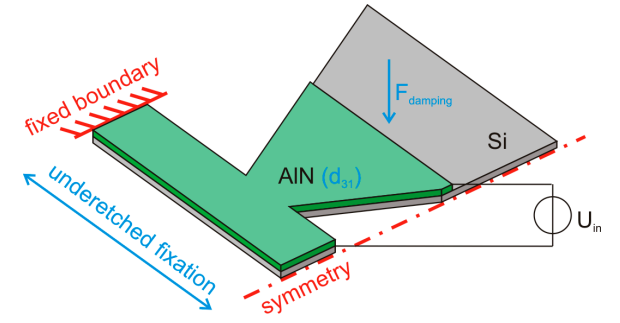

(a)

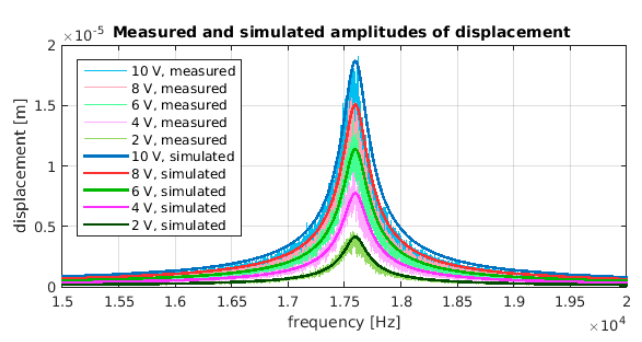

(b)

Figure 3. (a) Schematic view of the simulation model: The device is fixed at the left side and a symmetry boundary condition is applied at the right side. By applying an electrical field the AlN layer is strained in in-plane direction, which results in an out-of-plane bending of the flapper. The model parameters displayed in blue font are calibrated using LDV measurements; (b) Measurements and simulations of the frequency response of an exemplary twin-fin flapper, applying damping forces extracted from measurements, depending on the input voltage.

\section{Fluidic Simulations}

In previous studies [4] we proved, that an undulatory motion can be induced by using two electrodes (as sketched in Figure 1b) instead of the here investigated one electrode design, when applying a phase shifted second input signal to the top-electrode at the rear part of the flapper. However, we observe a trade-off between the requested high deflection and the desired undulatory characteristics of the motion. This trade-off depends on the ratio of the actuation frequency $f$ and the eigenfrequency $f_{0}$. When $f / f_{0} \rightarrow 1$, the flapper oscillates in its first eigenmode, hence maximizing its amplitude of deflection, but moving simply up and down without any undulatory characteristic of the motion. In order to find the optimal compromise between these two requirements we carried out fluidic simulations of the fluid flow induced by a two dimensional bending actuator, which is positioned inside a micro channel. Turbulent and rotational flow simulations with moving fluid-solid interfaces are challenging for conventional finite element solvers. We were able to achieve very good results applying a finite volume instead of finite element method and an improved moving mesh algorithm to preserve a good mesh element quality during calculations.

This way, three different motion patterns are investigated (Figure 4a): pure resonant motion, pure undulation as it can be found in nature, and the pursued technological realization, i.e., a simplified undulatory motion which can be realized using the two-electrodes design with phase shifted actuation

Figure $4 \mathrm{~b}$ shows the expected increase of the volume flow at the outlet of the micro channel, when an undulatory motion is performed, which proves the efficacy of the proposed novel device concept. The loss of volume flow due to the simplification of the motion in comparison to pure undulation is acceptable. Furthermore the influence of the deflection amplitude is proven: Figure 4c shows a significant increase of volume flow, when the deflection is increased from $5 \mu \mathrm{m}$ to $25 \mu \mathrm{m}$, which as we know from the measurements of the prototypes, is a value that can be realized in future process runs by re-designing the device appropriately. 


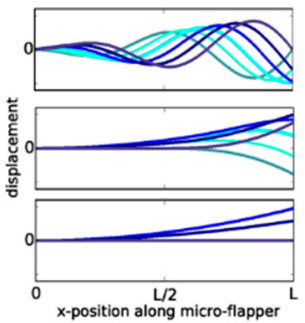

(a)

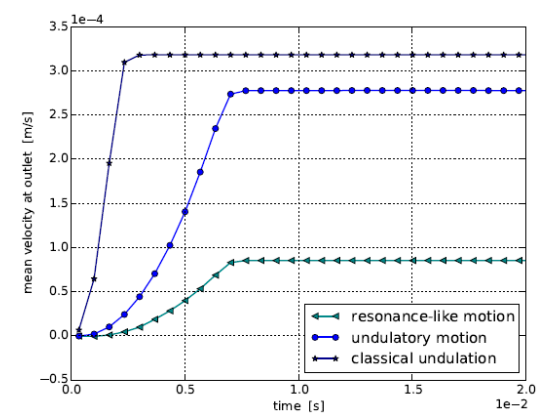

(b)

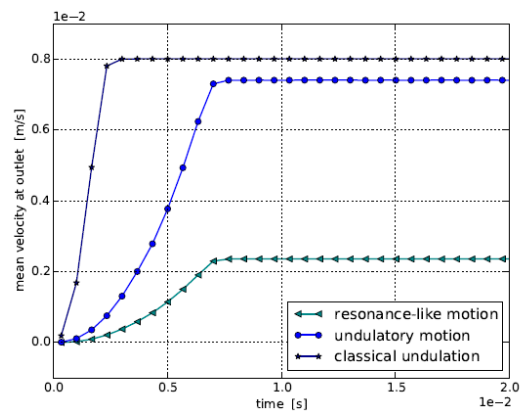

(c)

Figure 4. (a) Simulated motions of the flapper: Undulation with wavelike motion and increasing amplitude; undulatory motion reduced to the basic translatory und rotatory portion; resonant-like oscillation. $(\mathbf{b}, \mathbf{c})$ Time dependent volume flow at the outlet of the 2D micro channel for different amplitudes of deflection ((b) $5 \mu \mathrm{m}$ and (c) $25 \mu \mathrm{m}$ ).

In addition to the shape of the actuator's bending line and its amplitude of deflection, the actuation frequency has a major influence on the reachable volume flow. The logarithmic plot in Figure 5a shows, that an increase of some orders of magnitude can be achieved by adapting this frequency appropriately. This is due to the formation of a vortex wake as predicted from the natural archetype, as shown by an exemplary simulation result in Figure $5 b$.

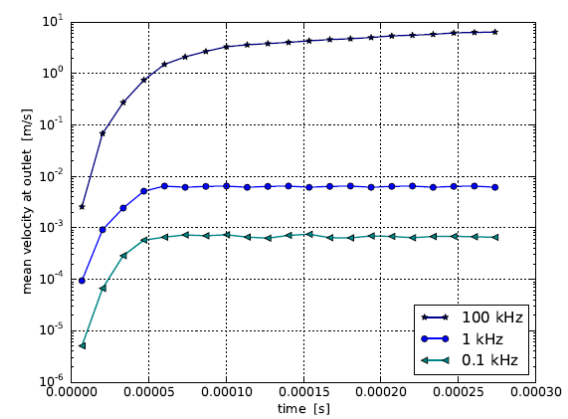

(a)

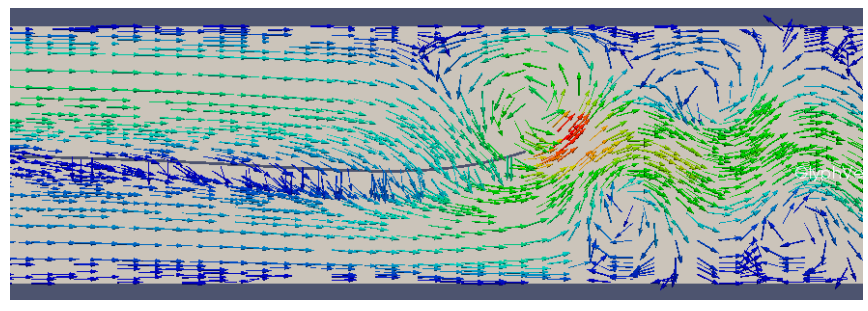

(b)

Figure 5. (a) Volume flow at the outlet of the micro channel for increasing oscillation frequencies; (b) Velocity field inside the micro channel induced by the flapping actuator: A vortex wake is generated by the undulatory motion of the device at $100 \mathrm{kHz}$, similar to the biological archetype. The superposition of the vortices creates an axial jet stream, hence a well-directed volume flow and good mixing behavior of the fluid inside the micro channel.

\section{Conclusions and Next Steps}

We presented prototypes of a novel fluidic actuator conceived and designed for cooling and venting applications. They show promising high amplitudes of deflection and are used to calibrate the piezoelectric mechanical simulation model which is needed for re-design and optimization of the devices. Especially the knowledge of the occurring damping force is important to evaluate and compare new design variants on the basis of reliable simulations of their operation. By extensive fluidic simulations we could prove that the natural archetype can be emulated and the induced volume flow can be significantly enhanced by choosing the proper actuation regime of the actuator. These first investigations are the basis for further optimizations aiming at the best trade-off between actuation near resonant frequency (hence maximum deflection) and undulatory actuation (maximum directed fluid flow).

Extensive parameter studies w.r.t. these aspects are planned in order to initiate an optimized redesign of the devices as well as the characterization of the generated fluid velocity field using MicroParticle-Image-Velocimetry ( $\mu$ PIV). 


\section{References}

1. Lighthill, J. Large-Amplitude elongated-Body Theory of Fish Locomotion. In Mathematical Biofluiddynamics; Society for Industrial and Applied Mathematics: Philadelphia, PA, USA, 1975.

2. Nachtigall, W.; Wisser, A. Insektenflug, Konstruktionsmorphologie, Biomechanik, Flugverhalten; Springer: Berlin/Heidelberg, Germany; New York, NY, USA, 2003.

3. Hampl, S.; Hoffmann, M. Piezoelectric AlN bimorph benders as low-frequent resonant micro-actuators with large travel range. In Proceedings of the Mikrosystemtechnik Kongress, Aachen, Germany, 23-25 October 2013.

4. Behlert, R.; Schrag, G.; Wachutka, G. Efficient Fluid Transport by a Bionically Inspired Micro-Flapper: Fluidic Investigations Using Fully Coupled Finite Element Simulation. In Proceedings of the SPIE Micro Technologies, Barcelona, Spain, 8 May 2017.

(C) 2017 by the authors. Licensee MDPI, Basel, Switzerland. This article is an open access article distributed under the terms and conditions of the Creative Commons Attribution (CC BY) license (http://creativecommons.org/licenses/by/4.0/). 